\title{
SMS over LTE: Interoperability between Legacy and Next Generation Networks
}

\author{
Filipe A. Leitão, Sérgio S. Freire \\ Department of Network Platforms and Multimedia Solutions \\ Portugal Telecom Inovação, SA, Aveiro, Portugal \\ Email: \{filipe-a-leitao,sergio-s-freire\}@ptinovacao.pt
}

\author{
Solange Rito Lima \\ Department of Informatics \\ University of Minho, Braga, Portugal \\ Email: solange@di.uminho.pt
}

\begin{abstract}
To increase the trust and acceptance of Next Generation Networks (NGN) by new and existing subscribers, it is necessary to ensure that the most popular services of legacy networks (GSM/GPRS) are still in place. The Short Message Service (SMS) is a widespread data service with high revenue for telecommunication operators, which allows the exchange of short messages between fixed, mobile and, indirectly, Internet subscribers.

In this context, it is essential to extend this service for the NGN and allow its interaction with the new messaging services. With that in mind, a study was carried out to ensure the coexistence between SMS and the Instant Messaging (IM) service present in NGN. The present study identifies the relevant technical specifications impacting on the functionality of the IP Multimedia Subsystem (IMS) messaging services so that the interoperability with SMS can be ensured.

The results of this study, covered in this article, aim to help the comprehension of the proposed interoperability solution, and demonstrate that the exchange of messages between legacy and NGN messaging services or equipment can be successfully accomplished. In this context, both SMS and IMS messaging services are detailed, along with the main protocols they involve. Finally, the entity responsible for ensuring message interoperability - the IP Short Message Gateway (IP-SM-GW) is presented and tested in a commercial delivery content testbed.
\end{abstract}

\section{INTRODUCTION}

As the evolution to Next Generation Networks (NGN) seems inevitable for mobile operators, a lot of issues come around concerning the support of legacy services by Long Term Evolution (LTE) networks [1]. One of the most important service to support is definitively the Short Message Service (SMS).

The SMS firstly appears in the Global System for Mobile communications (GSM) standardization by the European Telecommunications Standards Institute (ETSI) as a notification system for voice mail messages based on the old paging system. Today, the SMS is supported by the 3rd Generation Partnership Project (3GPP) [2], and is one of the most popular mobile services, as a voice call alternative, with users exchanging millions of text messages every day. Even with modern and appealing services offered by the telecommunications operators (mostly data services), SMS usage has grown continuously in the last few years, and that is a tendency to maintain. For those reasons, this service represents great part of operators revenue, which they cannot afford to loose [3], [4].

The main concern of the mobile operators about this legacy messaging support in NGN is because SMS is not only about text. Most of today's mobile services are heavilydependent on SMS for internal operations, remote device configurations and information procedures, such as roaming updates. Additionally, mobile operators want to get revenue from previous investments in commercial services such as message broadcasting, direct advertising and other value-added services that rely on SMS.

Abandoning a Circuit Switched (CS) architecture and migrate to an all-IP NGN such as IP Multimedia Subsystem (IMS), also supported by the 3GPP [5], it is not an easy task for telecommunications operators. In fact, this has been an hot topic on the latest meetings of standardization entities, where operators try to achieve a consensus for the CS fallback and evolution of SMS for LTE networks standardization [6].

All these issues around CS fallback and legacy services support in LTE have raised doubts about the SMS support in this NGN. That support is indeed possible despite all issues or medium-term costs that may be involved. In most developed countries, which already have a mature mobile network infrastructure, it is not possible to deploy all services on LTE since day one. This migration has to be progressive to minimize the impact on the mobile operators revenue. Thus, two distinct scenarios can be considered: a transition scenario where legacy networks will live side by side with NGN; and a full all-IP network scenario. In this article, we will focus on the transitional scenario.

It is in this context that the 3GPP proposed in its release 7 SMS support for IMS networks, presenting the IP Short Message Gateway (IP-SM-GW) [7]. This is a gateway that provides protocol interoperability between the SMS and the IMS messaging services. In this article, we describe the IPSM-GW and its major functionality. It is discussed how the Short Message can be delivered in an IMS environment at two levels: Transport-Level; and Service-Level. To provide a proof-of-concept, we present a testbed that was developed to assure the delivery of commercial content for GSM in an IP architecture using the IP-SM-GW. Clarifying and synthesizing the most relevant technical specifications on this subject and demonstrating that the exchange of messages between 
legacy and NGN messaging entities can be successfully accomplished, we expect to contribute for the comprehension of the proposed interoperability solution and foster NGN deployment.

This paper is organized as follows: related messaging services are presented in Section II; the solution for the messaging services interoperability is explained in Section III; the testbed environment is presented in Section IV; and, finally, some final comments are provided in Section V.

\section{Two Distinct Messaging Services}

\section{A. The Short Message Service}

The SMS is deeply dependent on the CS architecture. Its store-and-forward nature is built under the Signaling System \#7 (SS7) of GSM. The service messages are deployed by Mobile Application Part (MAP) messages [8], which are exchanged by the GSM infrastructure entities into two major procedures: (i) the Short Message Mobile Originated (SMMO), for Short Message Entity (SME) messages submission to the SMS Center (SMS-C); and (ii) the Short Message Mobile Terminated (SM-MT), for messages delivery from the SMS-C to the SME [2]. For each procedure, there are several MAP signaling messages in the GSM architecture concerning routing information, for the message delivery, or mobile device notifications, for instance to exchange the service status (device capacity full or device capacity available again to receive new messages).

Although the main purpose of this service is exchanging text between GSM terminals, nowadays the transport of binary payloads has been explored for commercial purposes such as the delivery of images and ringtones. Another relevant use of SMS, done by the network operators themselves, is to remotely configure terminals or to send important notifications related to users' billing plans.

The payload of a Short Message is 140 bytes long and, depending on the encoding scheme, one message can carry out till 160 characters. However, the SMS supports concatenated messages which allows to send several Short Messages that will be considered as a single one [2], [4].

\section{B. IMS Messaging Protocols}

The IMS architecture supports a large number of services that operate under the signaling protocol Session Initiation Protocol (SIP). Those services are mostly data services that can be accessed anywhere by an IP terminal. The IMS service offering includes, obviously, the messaging services.

The existing messaging services in IMS have been divided into two concepts: Page-Mode Messaging; and Session-Mode Messaging [5].

1) Page-Mode Messaging: This concept is similar to SMS, where a message is sent outside the context of a conversation, i.e., the message is sent without requiring an answer. It can be a notification, a single text message from a user to another, or even a way to send a media file. This concept suggests two types of messages: Short and Large messages [9].
A Short message in IMS can be sent using the SIP MESSAGE method [10]. This type of message can carry out text in its body or any other MIME type object, as shown in Figure 1. However, this method has SIP protocol limitations regarding message size. For congestion control purposes, a SIP message cannot exceed the size of 1500 bytes [11].

When an IMS user agent wants to send a message that will exceed the size limitation of the SIP protocol, that message is considered a Large message and the Message Session Relay Protocol (MSRP) [12] is used. Thus, Large messages may be used to send media objects over an IMS network. In the Page-Mode messaging context, the MSRP protocol is used for delivering only one message. This message can, however, be divided into various MSRP messages, as illustrated in Figure 2 , and merged in the destination entity as they all have the same identifier.

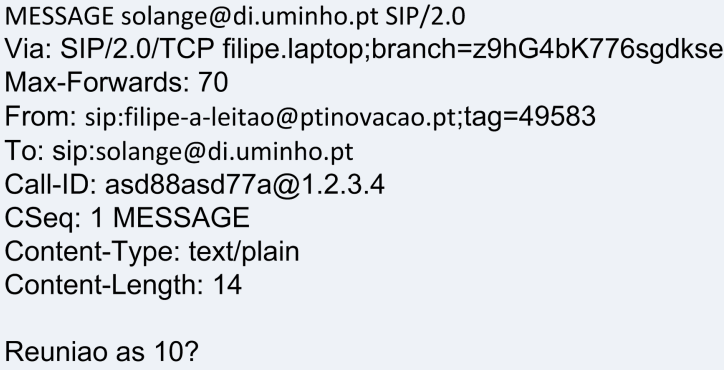

Fig. 1: SIP MESSAGE method

To deliver a MSRP message, a SIP session has to be established. Figure 3 details the establishment of a MSRP session. When all the message chunks are received, the session is closed without waiting for any response from the destination entity.

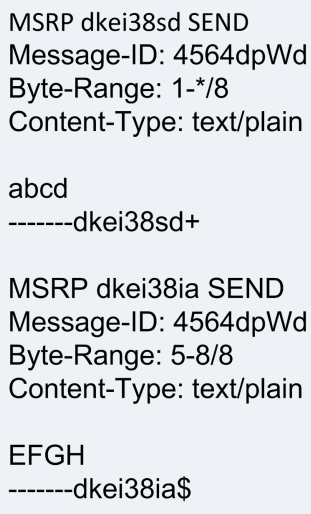

Fig. 2: Text message divided in two MSRP chunks

2) Session-Mode Messaging: This is the most common method for messages exchange between IMS users, and it is usually related with Instant Messaging (IM). In this method, 
a session is established for conversation between two or more IMS users.

For this type of messaging, the MSRP protocol is used in the IMS network side. However, unlike in the Page-Mode messaging, the MSRP session will be maintained until the end of the conversation.

3) OMA SIMPLE IM: Based on the concepts mentioned before, and the approaches developed by the IETF working group SIMPLE [13], the Open Mobile Alliance (OMA) has released an IM service for IMS called the OMA SIMPLE IM [14]. This service defines a set of operations deploying a messaging service in IMS, according to 3GPP messaging standards documentation [7].

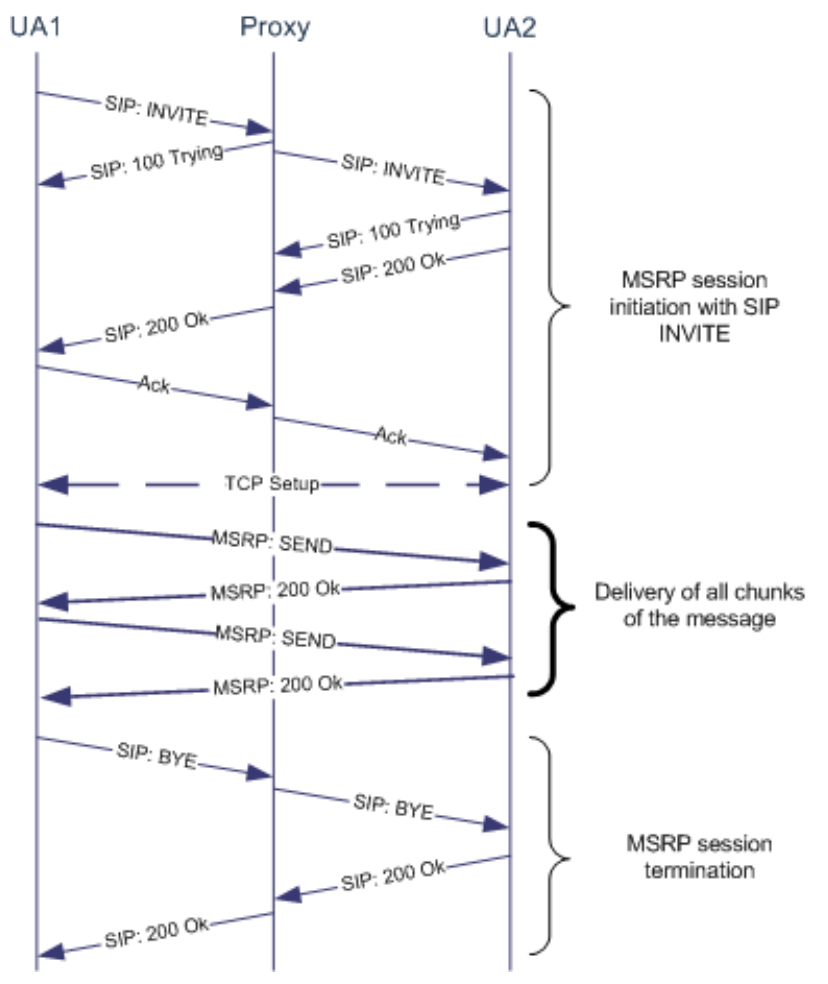

Fig. 3: Page-Mode messaging with MSRP

\section{SMS OVER IP}

The IMS is an all-IP architecture that emerged from 3GPP release 5 to comply with NGN prerequisites [15]. At its creation no native support for SMS was provided, for obvious reasons. The 140 bytes of the Short Message make no sense in an Internet Protocol (IP) network. Thus, IMS uses SIP for its signaling procedures, which is far different from the old MAP used in GSM [11], [16].

For several reasons, mentioned earlier, interoperability between the legacy service (SMS) and the new messaging services of IMS is essential for the acceptance of this network. The IP Short Message Gateway aims to sustain this integration.

\section{A. The IP Short Message Gateway}

In the 3GPP release 7, a gateway for IMS network was introduced: the IP Short Message Gateway (IP-SM-GW). This gateway's functionality assures the protocol interoperability between the SMS and the IMS messaging services.

The IP-SM-GW is placed between the core network of IMS and GSM. This gateway corresponds to an Application Server in the IMS architecture, which will be seen as a Mobile Switching Center (MSC) in the GSM network. To play its role in both architectures, the IP-SM-GW will be provided with the respective interfaces. This means MAP support in E/Gd interface, and SIP support in ISC interface. Additionally, the IP-SM-GW will be connected with the network operators database Home Subscriber Service (HSS) using Sh (Diameter protocol [17]) and J (MAP) interfaces, and with the charging domain using Ro and Rf interfaces [18], which are both Diameter protocol interfaces. Figure 4 resumes the IP-SM-GW environment.

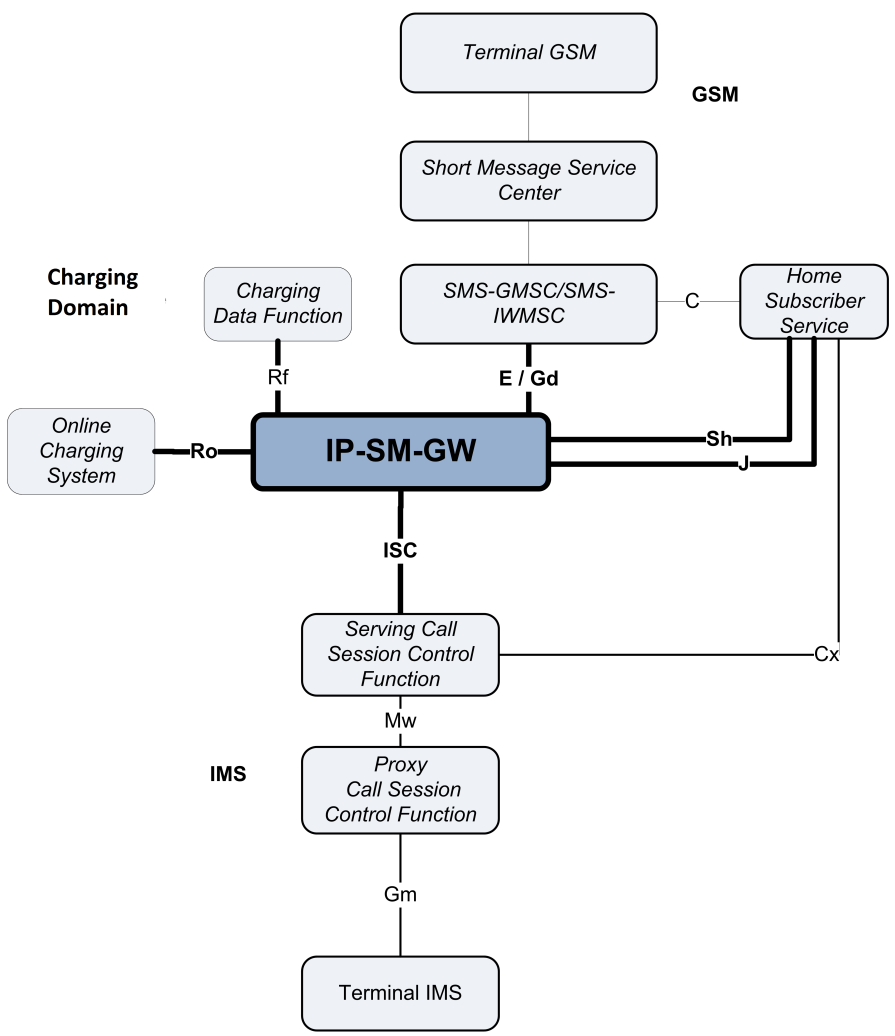

Fig. 4: The IP-SM-GW architecture as suggested by the $3 \mathrm{GPP}$ TS 23.204 [7].

This gateway is not only a protocol translator. It brings an additional complexity to the message treatment because it has to support all functionality of the covered messaging services. When a Short Message arrives from the GSM domain for an user in the IMS domain, the IP-SM-GW will check for his preferences and SMS device support to choose the delivery method. The same occurs in the reverse situation, when an IMS message has to be delivered in the GSM network. 
To solve this problem, two interoperability methods have been standardized by the 3GPP: the Transport-Level interworking [19]; and the Service-Level interworking [20].

\section{B. Transport-Level Interworking}

In Transport-Level interworking, the original SMS content is brought to the IMS side. The difference on this network relies on the transport protocol to be used to carry the Short Message, as all the SMS procedures [2] are maintained, as shown in Figure 5.

When the IP-SM-GW chooses to apply this method to forward a GSM message to the IMS domain, it just uploads the binary payload of the received MAP message into a SIP message. This message will use the SIP MESSAGE method and his payload will be signalled as 'application/vnd.3gpp.sms' [19]. Figure 6 exemplifies this type of messaging in IMS.

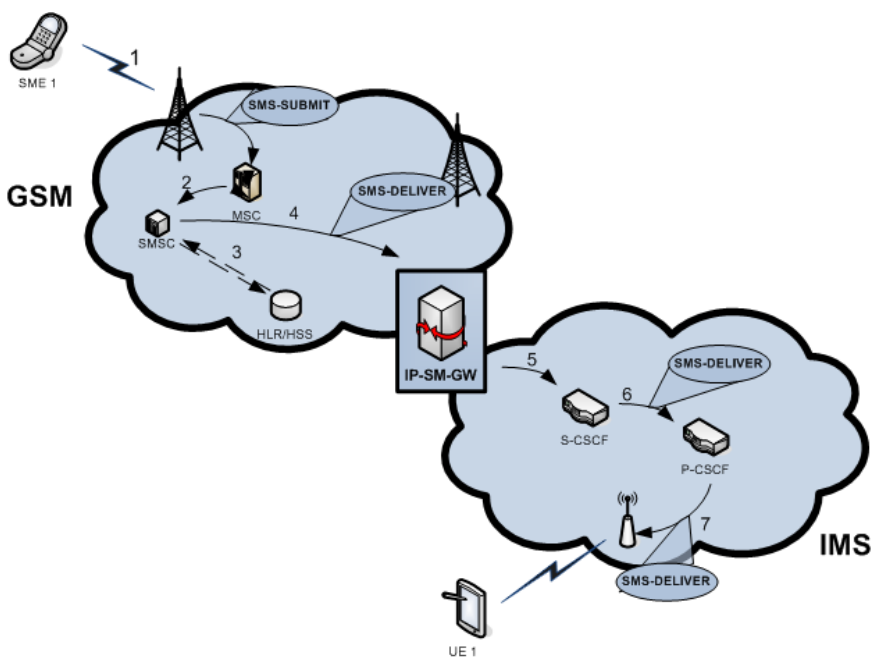

Fig. 5: Short Message delivery with Transport-Level interworking [7].

This method requires that the IMS destination entity supports the SMS payload of the SIP MESSAGE. This is the case when the destination of the Short Message is a smartphone with SMS support, or even a legacy service operating in the new domain. However, the communication with terminals without SMS support is also covered by the IP-SM-GW.

\section{Service-Level Interworking}

The Service-Level interworking is used whenever an IMS entity does not support SMS and needs to send/receive a Short Message. When IP-SM-GW detects this situation, by analyzing the destination entity preferences, it will make a full translation of the related messaging protocols. This means that, when receiving a Short Message from a legacy domain, the gateway uploads the Short Message's payload assuring the compliance with the destination's messaging support. Usually, in IMS, this requires translating the Short Message payload
MESSAGE sip:+351966666666@ims.ptinovacao.pt SIP/2.0 Via: SIP/2.0/UDP sip.ims.ptinovacao.pt From: < sip:+351966123123@ims.ptinovacao.pt>tag=14143 To: <sip:+351966666666@ims.ptinovacao.pt> Call-ID: apb03a0s09dkjdfglkj49232 Max-Forwards: 70

Allow: INVITE,ACK,CANCEL,BYE,MESSAGE,OPTIONS,NOTIFY,PRACK,UPDATE,REFER User-Agent: Content-Type: application/vnd.3gpp.sms

Authorization: Digest username="filipe-a-leitao@ptinovacao.pt",realm="ims.ptinovacao.pt",nonce="',uri="sip:ims.ptinovacao.pt" P-Preferred-Identity: < sip:+351966123123@ims.ptinovacao.pt> Allow-Events: presence, presence.winfo

0000000691330100000F320691330100000F11000B9133163254760000BB65F330BB4E07

Fig. 6: SIP MESSAGE carrying a Short Message payload.

to a plain/text payload of an Instant Message, as presented in Figure 1.

When an Instant Message needs to be sent to the legacy domain, the IP-SM-GW can undertake two distinct actions: translate the message into a Short Message and forward it to the Short Message Service Center (SMSC) as a regular submission procedure; or translate and deliver the message directly to the destination entity. In the latter, we can consider that the IMS Instant Messaging service has been extended to the legacy domain.

One major concern about using the Service-Level interworking is the SMS signalling procedures without direct relation with the new domain's messaging services. This is the case of delivery reports; since the moment that the message enters the new domain, the sender looses its. To solve this problem, the SIP message's payload can be encoded using the Common Profile for Instant Messaging (CPIM) protocol [21], which allows to use the Instant Message Disposition Notification (IMDN) header [22]. This header provides four types of notifications about an Instant Message state: delivered; not delivered; processed; and visualized. An example of the structure of this message can be seen in Figure 7.

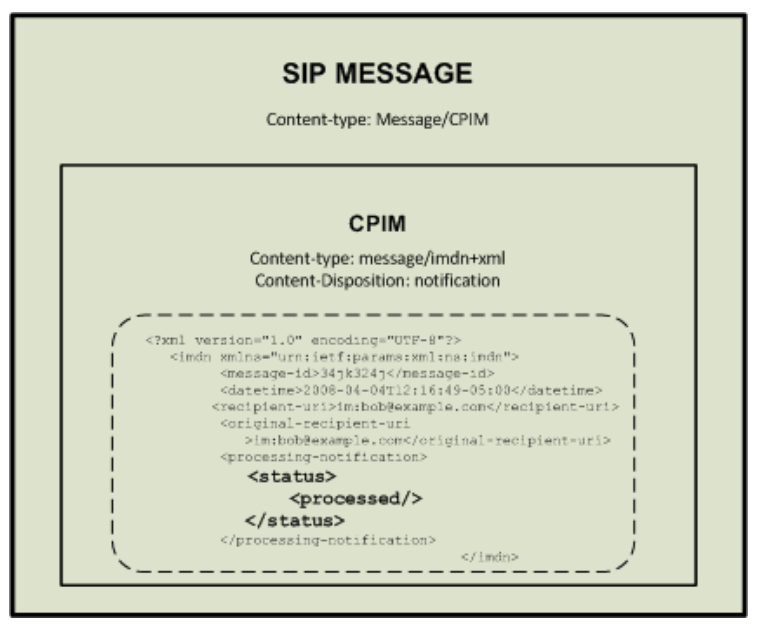

Fig. 7: IMDN for a processed message.

This payload encapsulation allows to request a delivery 


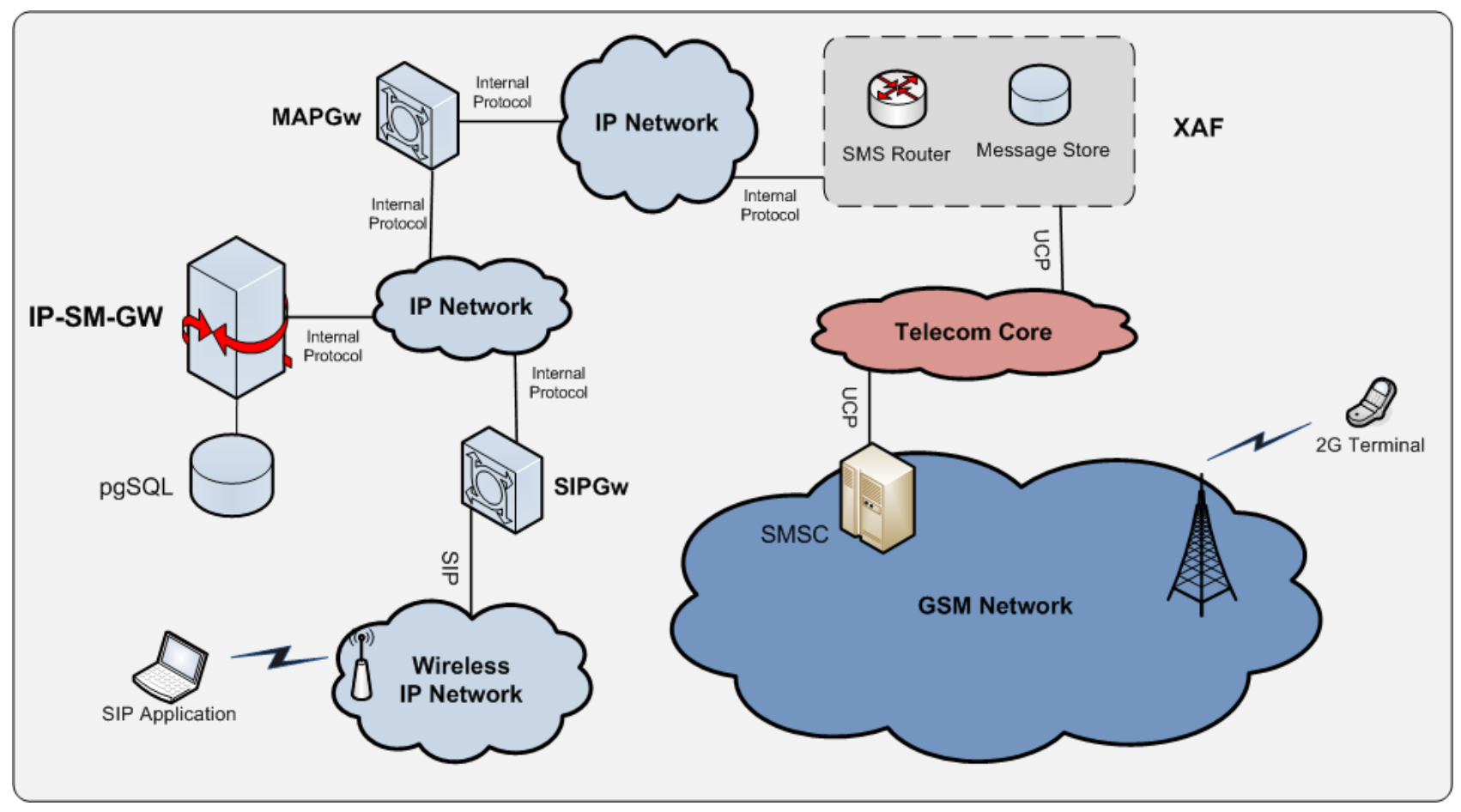

Fig. 8: Created Testbed.

notification for a Short Message delivered as an Instant Message. With the IP-SM-GW controlling and tracking the delivery state, a Short Message delivery report can easily be generated and sent back to the SMSC, concerning an previously received and forwarded Short Message.

\section{Delivering Content in IMS From Legacy SERVICES}

One major concern of telecommunications operators about moving forward into an IMS implementation are the previous investments in legacy infrastructures. An important example is undoubtedly SMS exploration for commercial purposes. Here we can include value-added services, media content requests and broadcast services, among others. Those services represent a huge part of operators revenue that they cannot afford to loose. Additionally, services such as Short Messages broadcast are largely used by institutional entities, as stated during the latest pandemic flu, where governments have used this service to broadcast alerts to the population. More, value-added service providers or even end users are mostly indifferent to changes happening in the network side; imposing different interfaces to the clients is not a viable option. Therefore, the implementation of an IP-SM-GW is of major importance while legacy services are still in place.

In this context, in order to evaluate the functionality of the IP-SM-GW, a testbed has been deployed including an implementation of the IP-SM-GW using JAVA technology. This testbed is detailed below.

\section{A. Testbed}

Figure 8 illustrates the relevant entities regarding the evaluation of IP-SM-GW functionality. To simulate an IMS network, a small SIP network has been created. Nevertheless, the core of IP-SM-GW is fully prepared for a real IMS network.

As mentioned, the IP-SM-GW core was developed using JAVA technology. The logical core is supported by a PostgreSQL database, which is responsible for keeping user's data (such as routing and delivery preferences), the messages being delivered and internal configuration parameters.

In the setup of this testbed, the logical core development has been separated from the network interfaces development. Thus, the IP-SM-GW core has been created having a real IMS implementation in mind, and still be able to use a SIP gateway (SIPGw) to evaluate its functionality. The SIPGw was also developed in JAVA using the JAIN SIP stack [23].

The GSM interface has been created as a MAP gateway (MAPGw). In this testbed, as shown in Figure 8, the MAPGw is the interface between the IP-SM-GW and a SMS commercial content platform (called XAF). This platform was merged to this testbed for two reasons: to test the IP-SM-GW behavior for the Short Messages broadcast service; and to facilitate the IP-SM-GW integration in a real GSM core network, outside our control.

\section{B. Results}

On the created testbed, two types of tests were carried out: Short Messages broadcasts; and point-to-point communication. This article presents results from this latter test. 
In this test, an Instant Message from the SIP softphone is sent to a GSM terminal. Figure 9 shows the SIP application used to generate the SIP message. The intention is to send the message for the GSM terminal using the MSISDN 351966000001 .

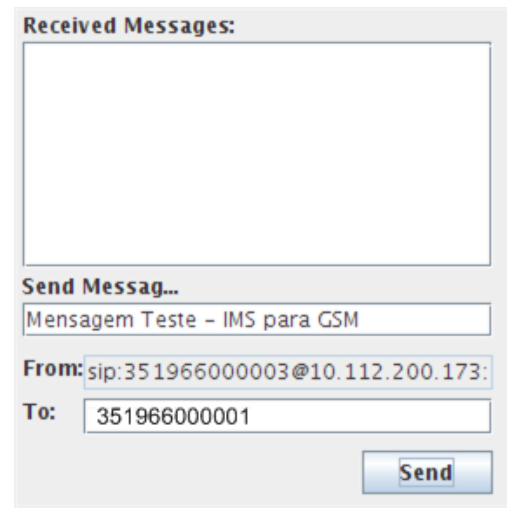

Fig. 9: SIP application front end.

This application builds a SIP MESSAGE and sends it to the IP-SM-GW through the SIPGw. After receiving the message, the IP-SM-GW will then generate an internal message and submit it to the SMSC using the MAPGw and the XAF platform.

At this point, the submitted message is seen as a regular Short Message from the SMSC point-of-view, and it can be delivered as a single message. Figure 10 shows how the received message looks like in a regular GSM phone.

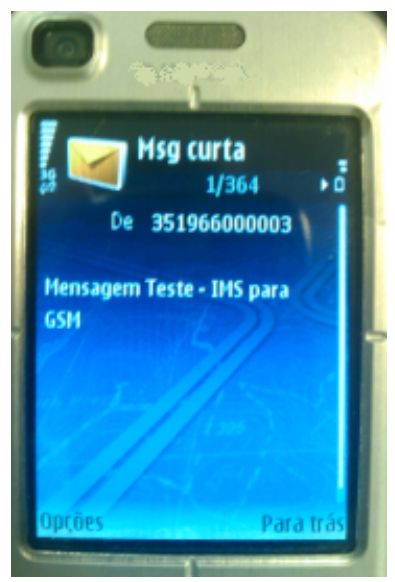

Fig. 10: Received Short Message.

\section{CONCLUSION}

IMS implementations are currently a reality for most telecommunications operators. However, the business broadness of these implementations could be enlarged if legacy services coexist in the new domain. On the other hand, the migration of users to NGN could be faster if their favorite services in legacy networks were still present. This is the case of SMS.

The IP-SM-GW is undoubtedly an essential component for accomplishing a faster and smoother migration of the legacy messaging services to the IMS network.

On this article, the technology for IMS messaging has been explained, and the solutions to integrate SMS into this network have been detailed. A testbed for testing IP-SM-GW functionality has been developed; the initial interoperability tests carried out have proved that the IP-SM-GW can provide a seamless solution for SMS support in LTE networks.

\section{REFERENCES}

[1] E. Dahlman, S. Parkvall, S. Skold, and P. Beming. 3G Evolution: HSPA and LTE for Mobile Broadband. Academic Press, second edition, 2008.

[2] 3GPP. Technical realization of Short Message Service (SMS). TS 23.040, 3rd Generation Partnership Project (3GPP), September 2008.

[3] Portio Research Limited. Mobile Messaging Futures 2009-20013. Nov. 2007.

[4] J. Brown, B. Shipman, and R Vetter. SMS: The Short Message Service. Computer, 40(12):106-110, Dec. 2007.

[5] 3GPP. IP Multimedia Subsystem (IMS); Stage 2. TS 23.228, 3rd Generation Partnership Project (3GPP), September 2008.

[6] 3GPP TSG SA nr44. SMS over LTE. SP 090429, 3rd Generation Partnership Project (3GPP), June 2009.

[7] 3GPP. Support of Short Message Service (SMS) over generic 3GPP Internet Protocol (IP) access; Stage 2. TS 23.204, 3rd Generation Partnership Project (3GPP), September 2008.

[8] 3GPP. Mobile Application Part (MAP) specification. TS 29.002, 3rd Generation Partnership Project (3GPP), September 2008.

[9] 3GPP. IP Multimedia Subsystem (IMS) messaging. TS 23.240, 3rd Generation Partnership Project (3GPP), December 2009.

[10] B. Campbell, J. Rosenberg, H. Schulzrinne, C. Huitema, and D . Gurle. Session Initiation Protocol (SIP) Extension for Instant Messaging. RFC 3428, Internet Engineering Task Force, December 2002.

[11] J. Rosenberg, H. Schulzrinne, G. Camarillo, A. Johnston, J. Peterson, R. Sparks, M. Handley, and E. Schooler. SIP: Session Initiation Protocol. RFC 3261, Internet Engineering Task Force, June 2002.

[12] B. Campbell, R. Mahy, and C. Jennings. The Message Session Relay Protocol (MSRP). RFC 4975, Internet Engineering Task Force, September 2007.

[13] J. Rosenberg. SIMPLE made Simple: An Overview of the IETF Specifications for Instant Messaging and Presence using the Session Initiation Protocol (SIP). Internet-Draft draft-ietf-simple-simple-03, Internet Engineering Task Force, July 2008. Work in progress.

[14] Open Mobile Alliance. OMA SIMPLE IM V1.0, 2008.

[15] ITU-T. General Overview of NGN, December 2004.

[16] Miguel-Angel Garcia-Martin Gonzalo Camarillo. The 3G IP Multimedia Subsystem (IMS): Merging the Internet and the Ce llular Worlds. Wiley \& Sons, second edition, 2004.

[17] P. Calhoun, J. Loughney, E. Guttman, G. Zorn, and J. Arkko. Diameter Base Protocol. RFC 3588, Internet Engineering Task Force, September 2003.

[18] 3GPP. Telecommunication management; Charging management; Short Message Service (SMS) charging. TS 32.274, 3rd Generation Partnership Project (3GPP), 2008.

[19] 3GPP. Support of SMS over IP networks; Stage 3. TS 24.341, 3rd Generation Partnership Project (3GPP), December 2008.

[20] 3GPP. Service Level Interworking for Messaging Services; Stage 3. TS 29.311, 3rd Generation Partnership Project (3GPP), September 2008.

[21] J. Peterson. Common Profile for Instant Messaging (CPIM). RFC 3860, Internet Engineering Task Force, August 2004.

[22] E. Burger and H. Khartabil. Instant Message Disposition Notification (IMDN). RFC 5438, Internet Engineering Task Force, February 2009.

[23] M. Ranganathan and Phelim O'Doherty. JAIN-SIP: Java API for SIP Signaling, https://jain-sip.dev.java.net/ 